enhancement of the $\mathrm{T}$-d8 concentration relative to $\mathrm{EtOH}$ (bulk solution has a value of $\approx 0.25$ ) followed by a near-linear decrease after the break. Returning to the Fickian solution and using the slower turn on of the surface concentration could represent the $\mathrm{EtOH}$ diffusion into the polystyrene. Figure 9 with $\alpha=1$ would be indicative if an induction period was included. That the EtOH is constrained from significantly penetrating the polystyrene until it has been modified by the T-d8 could be understood in terms of wetting phenomena. This would lower the EtOH concentration at the surface and give rise to a delay in the buildup of the EtOH concentration.

In general these experiments show no significant evidence for simple Case II diffusion behavior. There may be several reasons for this result. The distance scale is significantly less than that used in most sorption experiments. The measurements are done in "real time". A true three-component chemical system has been studied in contrast to many studies that have two components and a "marker" component that is treated as an inert material. And, finally, the fused silica substrate may introduce some compensating strain into the polymer. Some of these questions will be addressed in future work.

\section{Conclusions}

It has been demonstrated that it is possible to monitor all the chemical species involved in a diffusion process and that it is important to do so. Waveguide Raman sampling allows real time sampling to be done and is applicable to many polymer/solvent systems and potentially polymer/polymer systems. The assumption that the initial materials and concentrations remain unchanged is not valid in this case and probably questionable in most cases of chemical diffusion. The length scale for this experiment was on the order of $1 \mu \mathrm{m}$ with submicrometer resolution possible. Although the results indicate that the solvents EtOH and $\mathrm{T}-\mathrm{d} 8$ are controlled by different parameters, it is probably not possible to decide what model of diffusion is correct. Even further work to quantify these results may not be able to separate the models due to their empirical and adjustable natures. What does seem clear from these results and those in the literature is that molecular interactions must be incorporated from the start and that a microscopic model of diffusion is needed.

Registry No. EtOH, 64-17-5; polystyrene, 9003-53-6; toluene- $d_{8}$, 2037-26-5.

\title{
Primary Electron Transfer in Photosynthetlc Reaction Centers
}

\author{
Eberhard v. Kitzing and Hans Kuhn* \\ Max-Planck-Institut für biophysikalische Chemie, Am Fassberg, Göttingen, F.R.G. (Received: April 26, 1989)
}

\begin{abstract}
A simple model is presented for the primary step in the photoinduced electron transfer in the photosynthetic reaction centers of Rps. viridis and $R b$. sphaeroides. The interaction of the chromophore system (consisting of photoexcited donor $P$, conduction intermediate $B_{L}$, and acceptor $H_{L}$ ) with the environment is assumed to be negligible until vibronic deexcitation takes place resulting in a stochastically perturbed adiabatic electron transfer. This process constitutes a three-level problem. It is shown that this problem, in the present case, can be approximated by a two-level problem which can simply be solved. The energy level of $\left|\mathrm{B}_{\mathrm{L}}{ }^{-}\right\rangle$is found to be $0.06 \mathrm{eV}$ above the level of $\left|\mathrm{P}^{*}\right\rangle$. The unidirectionality of the electron flow is explained by coincidence of energy levels in the $\mathrm{L}$ branch due to evolutionary constraint.
\end{abstract}

\section{Introduction}

In the primary electron-transfer step in the photosynthetic reaction center of purple bacteria the electron donor $P$, the special pair, a bacteriochlorophyll dimer, is photoexcited to $\mathrm{P}^{*}$ and the electron is transferred within some picoseconds from $P^{*}$ (via $B_{L}$ the accessory bacteriochlorophyll) to the spectroscopically resolvable intermediate electron acceptor $\mathrm{H}_{\mathrm{L}}$ a bacteriopheophytin (for a recent review see ref 1 ). Fleming et al. ${ }^{2}$ have found an increase in electron-transfer rate $k$ with decreasing temperature which is stronger in Rps. viridis than in $R b$. sphaeroides. The rate of electron transfer from $P^{*}$ via $B_{L}$ to $H_{L}$ along the $L$-branch is at least 10 times larger than from $P$ via $B_{M}$ to $\mathrm{HM}_{M}{ }^{3}$ along the M-branch, which structure is nearly symmetric to that of the L-branch, and this leads to the unidirectionality of the charge separation.

The rate is of the order of a reciprocal picosecond at $10 \mathrm{~K}$. The electron transfer is given by a single rate constant, and a transient bleaching in the $B_{L}$ absorption region is absent. ${ }^{4}$ These findings

(1) Michel, H.; Deisenhofer, J. Bull. Inst. Pasteur 1988, 86, 37

(2) Fleming, G. R.; Martin, J. L.; Breton, J. Nature 1988, 333, 190

(3) Michel-Beyerle, M. E.; Plato, M.; Deisenhofer, J.; Michel, H.; Bixon, M.; Jortner, J. Biochim. Biophys. Acta 1988, 932, 52. Plato, M.; Möbius, K.: Michel-Beyerle, M. E.; Bixon, M.; Jortner, J. J. Am. Chem. Soc. 1988, 110,7279 .

(4) Breton, J.; Martin, J. L.; Fleming, G. R.; Lambry, J. C. Biochemistry $1988,27,8276$. are evidence against mechanisms ${ }^{5}$ in which $B_{L}^{-}$is a distinct, kinetically resolvable intermediary electron acceptor between $P^{*}$ and $\mathrm{H}_{\mathrm{L}}^{-}$.

It is difficult to rationalize the results on the basis of the conventional theory for nonadiabatic electron transfer. A nonadiabatic superexchange-mediated electron transfer from $\mathrm{P}^{*}$ via $B_{L}$ to $H_{L}$ has been proposed. ${ }^{3}$ The unidirectionality was explained as being due to a delicate difference in the electronic interaction terms between the two branches. ${ }^{3}$ The approach is based on the conventional assumption that the chromophores are strongly coupled to the environment, as described by the Marcus equation ${ }^{6}$ or its quantum mechanical analogues.

In the present approach the interaction with the environment is treated differently, and this leads to a different judgment of the role of specific structural asymmetries in inducing this unidirectionality. In contrast to the model in ref 3 , here the difference in energies of $\mathrm{H}_{\mathrm{L}}{ }^{-}$and $\mathrm{H}_{\mathrm{M}}{ }^{-}$is sufficient to explain the unidirectionality in electron transfer. The results by Fleming et al. ${ }^{2}$ can be rationalized. By its simplicity this approach should be of interest focusing on some important features. Studies on sitespecific mutants and on other organisms ${ }^{7}$ should shed light on the

(5) Shuvalov, V. A.; Amesz, J.; Duysens, L. N. M. Biochim. Biophys. Acta 1986, 851, 327. Marcus, R. A. Chem. Phys. Lett. 1987, 133, 471.

(6) Marcus, R. A. J. Chem. Phys. 1956, 24, 966.

(7) Yeates, T. O.; Komiya, H.; Chirino, A.; Rees, D. C.; Allen, J. P.; Feher, G. Proc. Natl. Acad. Sci. USA 1988, 85, 7993. 
role of the structural asymmetry and help in discriminating between models and in finding more refined models.

\section{Theory}

We consider a model which is based on the concept that there is negligible interaction of the chromophore system with the environment until a collision of vibronically excited $\mathrm{H}_{\mathrm{L}}{ }^{-}$is taking place (caused by random particularities in the transient environmental configuration). In this collision the vibrational energy is dissipated and the electron is trapped at $\mathrm{H}_{\mathrm{L}}$ in a time $(\approx 10 \mathrm{fs})$ short compared to the time between excitation and trapping $(\approx 1$ ps). This process can be considered as a stochastically perturbed adiabatic electron transfer.

The transfer matrix elements $\epsilon_{\mathrm{PB}}$ and $\epsilon_{\mathrm{BH}}$ for the electron transfers from $P^{*}$ to $B_{L}$ and from $B_{L}$ to $H_{L}$, respectively, were obtained by a quantum mechanical approximation. ${ }^{8}$ The values (being in the range between $5 \times 10^{-2}$ and $3 \times 10^{-3} \mathrm{eV}$ ) were found to depend very sensitively on details in the geometry. These values are based on free electron model wave functions. A refined treatment in which the amplitudes of the electrons in the considered orbitals were taken from INDO calculations and in which the long distance tail of the electronic wave function was calculated by a procedure proposed in ref 8 leads to values in the same range. We assume that $\epsilon_{\mathrm{PB}}$ and $\epsilon_{\mathrm{BH}}$ are in the above range and that the level of $\left|\mathrm{B}_{\mathrm{L}}^{-}\right\rangle$is by a certain amount $b$ higher than the level of $\left|P^{*}\right\rangle$, to take into consideration the fact that an intermediate $B_{L}{ }^{-}$ could not be spectroscopically identified. ${ }^{4}$

Furthermore, we assume at temperature $T=0$ that the vibronically excited level of $\left|\mathrm{H}_{\mathrm{L}}{ }^{-}\right\rangle(\hbar \omega \approx 0.17 \mathrm{eV}$ above ground-state level) coincides with the level of $\mathrm{P}^{*}$, and that the levels, by thermal noise, differ in the average, at temperature $T$ by $a= \pm^{1} /{ }_{2} k_{\mathrm{B}} T$, where $k_{\mathrm{B}}$ is Boltzmann's constant and $a$ is the amount by which the level of the vibronically excited system $\left|\mathrm{P}^{+} \mathrm{B}_{\mathrm{L}} \mathrm{H}_{\mathrm{L}}^{-}\right\rangle$is higher than the level of $\left|\mathrm{P}^{*} \mathrm{~B}_{\mathrm{L}} \mathrm{H}_{\mathrm{L}}\right\rangle$. Because of the strong electronic coupling (in contrast to the conventional case) the time until the phase disturbing collision is taking place is not short compared to the time of oscillation of the electron between $P$ and $H_{L}$.

For simplicity we first neglect $\mathrm{B}_{\mathrm{L}}$ and attribute the transfer matrix element $\epsilon$ to the transfer from $P^{*}$ to $H_{L}$. According to a well-known relation (see eq A.4 in the Appendix) the probability $p$ of finding the electron at $\mathrm{H}_{\mathrm{L}}$, in the average, is $p=1 / 2\left[(2 \epsilon)^{2} /\left(a^{2}\right.\right.$ $\left.\left.+(2 \epsilon)^{2}\right)\right]$. The rate is ( $\tau$ vibronic relaxation time)

$$
k_{0}=\frac{1}{2 \tau} \frac{(2 \epsilon)^{2}}{a^{2}+(2 \epsilon)^{2}}
$$

This relation is obtained by considering the probability of the electron to be trapped in $\mathrm{H}_{\mathrm{L}}$ during the time interval $\mathrm{d} t$. This probability $k_{0} \mathrm{~d} t$ equals the probability $p$ of the electron to be in $\mathrm{H}_{\mathrm{L}}$ times the probability $\tau^{-1} \mathrm{~d} t$ to be trapped when being in $\mathrm{H}_{\mathrm{L}}$; thus $k_{0}=p / \tau . k_{0}(a)$ gives the transfer rate at a certain $a$. In general the electronic levels will be slightly scattered around their average value by thermal motion. The standard deviation from the average is assumed to be $1 /{ }_{2} k_{\mathrm{B}} T$. The thermally averaged electron-transfer rate can be approximately given by

$k=\frac{1}{2 k_{\mathrm{B}} T} \int_{-k_{\mathrm{B}} T}^{+k_{\mathrm{B}} T} k_{0}(a) \mathrm{d} a=\frac{1}{2 \tau} \frac{2 \epsilon}{k_{\mathrm{B}} T} \arctan \left(\frac{k_{\mathrm{B}} T}{2 \epsilon}\right)$

In the considered range $k_{\mathrm{B}} T \leq 6 \epsilon$, this equation leads essentially to the same result as eq 1 with a given standard deviation of $\pm 1 /{ }_{2} k_{\mathrm{B}} T$.

In considering $\left|B_{\mathrm{L}}\right\rangle$ the three-state problem must be solved (instead of the two-state problem) which is quite complicated. However, in the case that the transfer matrix elements $\epsilon_{\mathrm{PB}}, \epsilon_{\mathrm{PH}}$, and $\epsilon_{\mathrm{BH}}$ are small compared to the energy differences of $b=E_{\mathrm{B}_{1}}$ $-E_{\mathrm{p}^{*}}$ and $b-a=E_{\mathrm{B}_{1}}-E_{\mathrm{H}_{1}}$, approximative perturbation methods can be used. As shown in the Appendix the three-level problem

(8) Kuhn, H. Phys. Rev. 1986, A34, 3409. Kuhn, H. Advances in Chemical Reaction Dynamics. In Proceedings of NATO Conference in Iraklion, 1985; Rentzepis, P., Capellos, Ch., Eds.; D. Reidel: Dordrecht, 1986; pp $525-550$

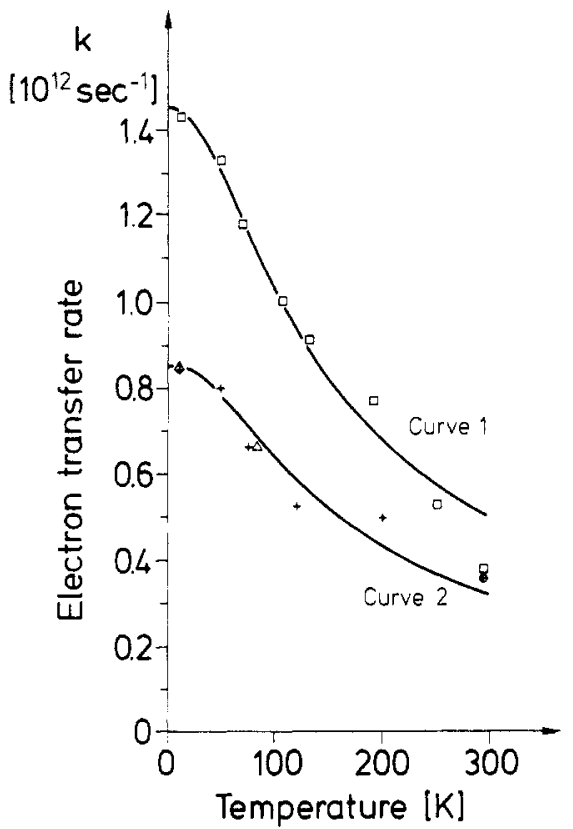

Figure 1. Rate of primary electron-transfer step as a function of temperature. Rps. viridis (ם); Rb. sphaeroides $(+)$ unmodified, (O), modified (removal of $\left.B_{M}\right) ;(\Delta)$ deuterated samples, according to ref 2 . Curve 1: eq la with $\epsilon=3.2 \times 10^{-3} \mathrm{eV}, \tau=3.4 \times 10^{-13} \mathrm{~s}$. Curve 2: eq 1a with $\epsilon=3.6 \times 10^{-3} \mathrm{eV}, \tau=5.9 \times 10^{-13} \mathrm{~s}$.

can be reduced to a two-level problem involving an effectivetransfer matrix element (Appendix, eq A.3)

$$
\epsilon=\epsilon_{\mathrm{PH}}+\epsilon_{\mathrm{PB}} \epsilon_{\mathrm{BH}}\left(\frac{1}{b}+\frac{1}{b-a}\right)
$$

where $\epsilon_{\mathrm{PH}}$ is the transfer matrix element for the direct transfer from $\mathrm{P}^{*}$ to $\mathrm{H}_{\mathrm{L}}$.

The transfer matrix elements $\epsilon_{\mathrm{PB}}, \epsilon_{\mathrm{PH}}$, and $\epsilon_{\mathrm{BH}}$ can be written as products of an electronic and a vibronic factor: $\epsilon_{i j}=\epsilon_{i j}{ }^{\text {el}}$. $\left\langle\phi_{i 0} \phi_{j 0} \mid \phi_{i v}^{\prime} \phi_{j w}^{\prime}\right\rangle$, where $\phi_{i 0}$ and $\phi_{j 0}$ are the vibrational wave functions of donor $i$ and acceptor $j$ before the electron transfer, $\phi_{i v}^{\prime}$ and $\phi_{j w}^{\prime}$ the corresponding functions after transfer, and $v$ and $w$ are vibrational quantum numbers of donor and acceptor, respectively. This approach is based on the simplifying assumption that a single normal vibration is excited. This can be justified; see ref 8. The vibrational energy is dissipated in $\left|\mathrm{P}^{+}\right\rangle$or in $\left|\mathrm{H}_{\mathrm{L}}{ }^{-}\right\rangle$ depending on whether the state where $\left|\mathrm{P}^{+}\right\rangle$is vibronically excited coincides with the original state or the state where $\left|\mathrm{H}_{\mathrm{L}}{ }^{-}\right\rangle$is vibronically excited.

In the first case $\epsilon_{\mathrm{PH}}=\epsilon_{\mathrm{PH}}^{\mathrm{el}}\left\langle\phi_{\mathrm{P} 0} \phi_{\mathrm{H} 0} \mid \phi_{\mathrm{P} 1}^{\prime} \phi_{\mathrm{H} 0}^{\prime}\right\rangle, \epsilon_{\mathrm{PB}}=\epsilon_{\mathrm{PB}}^{\mathrm{el}}$ $\left\langle\phi_{\mathrm{P} O} \phi_{\mathrm{BO}} \mid \phi_{\mathrm{P} 1}^{\prime} \phi_{\mathrm{B} O}^{\prime}\right\rangle$, and $\epsilon_{\mathrm{BH}}=\epsilon_{\mathrm{BH}}^{\mathrm{e}}\left\langle\phi_{\mathrm{B} 0} \phi_{\mathrm{H} O} \mid \phi_{\mathrm{B} O}^{\prime} \phi_{\mathrm{H} 0}^{\prime}\right\rangle$ and in the second case $\epsilon_{\mathrm{PH}}=\epsilon_{\mathrm{PH}}^{\mathrm{el}}\left\langle\phi_{\mathrm{PO}} \phi_{\mathrm{H} O} \mid \phi_{\mathrm{PO}}^{\prime} \phi_{\mathrm{H} 1}^{\prime}\right\rangle, \epsilon_{\mathrm{PB}}=\epsilon_{\mathrm{PB}}^{\mathrm{el}}\left\langle\phi_{\mathrm{PO}} \phi_{\mathrm{B} O} \mid \phi_{\mathrm{PO}}^{\prime} \phi_{\mathrm{B} O}^{\prime}\right\rangle$, and $\epsilon_{\mathrm{BH}}=\epsilon_{\mathrm{BH}}^{\mathrm{el}}\left\langle\phi_{\mathrm{B} O} \phi_{\mathrm{H} O} \mid \phi_{\mathrm{B} O}^{\prime} \phi_{\mathrm{H} 1}^{\prime}\right\rangle$. The vibronic terms are evaluated in ref 8 . In all cases a value of about 0.6 is obtained.

\section{Results and Discussion}

If $\epsilon_{\mathrm{PH}}$ is neglected and $b \gg a$ we obtain from eq 2 that $\epsilon=$ $2 \epsilon_{\mathrm{PB}} \epsilon_{\mathrm{BH}} / b$, and with $\epsilon_{\mathrm{PB}} \approx \epsilon_{\mathrm{BH}} \approx 10^{-2} \mathrm{eV}$ and $b=10^{-1} \mathrm{eV}$ this gives $\epsilon=2 \times 10^{-3} \mathrm{eV}$; this estimated $\epsilon$ value is in accord with the value of $\epsilon=3.2 \times 10^{-3} \mathrm{eV}$ obtained from experimental data (see below). The estimated values of $\epsilon_{\mathrm{PB}}$ and $\epsilon_{\mathrm{BH}}$ are within the range given above and the value of $b$ is in accord with the finding (see Note Added in Proof) that the probability $P_{\mathrm{B}_{\mathrm{L}}}$ of finding the electron in $B_{L}$ during the transfer process is about $16 \%$. In our model

$$
P_{\mathrm{B}_{\mathrm{L}}}=\frac{1}{2}\left[\left(\frac{2 \epsilon_{\mathrm{PB}}}{b}\right)^{2}+\left(\frac{2 \epsilon_{\mathrm{BH}}}{b-a}\right)^{2}\right]
$$

(see Appendix, eq A.5) and with $\epsilon_{\mathrm{PB}}=\epsilon_{\mathrm{BH}}=(b \epsilon / 2)^{1 / 2}$ and $a=$ 0 we find $P_{\mathrm{B}_{\mathrm{L}}} \approx 2 \epsilon / \mathrm{b}$. With $\epsilon=3.2 \times 10^{-3} \mathrm{eV}$ and $P_{\mathrm{B}_{\mathrm{L}}}=0.16$ this gives $b=0.04 \mathrm{eV}$. If we set $\epsilon_{\mathrm{BH}}=3 \epsilon_{\mathrm{PB}}$ (as suggested from 
INDO calculation) instead of $\epsilon_{\mathrm{BH}}=\epsilon_{\mathrm{PB}}$ the slightly different value $b=0.06 \mathrm{eV}$ is obtained.

With the above value $\epsilon=3.2 \times 10^{-3} \mathrm{eV}$, and with $\tau=3.4 \times$ $10^{-13} \mathrm{~s}$ curve 1 in Figure 1 is obtained from eq $1 \mathrm{a}$, in agreement with experimental points given by Fleming et al. for Rps. viridis. ${ }^{2}$ If we set $\epsilon=3.6 \times 10^{-3} \mathrm{eV}$ and $\tau=5.9 \times 10^{-13} \mathrm{~s}$ curve 2 is obtained, in agreement with the data observed for $R b$. sphaeroides. $^{2}$ The slight differences in $\epsilon$ and $\tau$ are reasonable, considering the sensitivity of $\epsilon$ on small changes in geometry and the dependence of relaxation processes on structural details.

We have assumed that the state where $\mathrm{P}^{+}\left(\right.$or $\mathrm{H}_{\mathrm{L}}{ }^{-}$) is vibronically excited coincides with the original state. The possibility that both states coincide with the original state can also be given. Then $k$ is the sum of two about equal terms and thus $\tau$ is to be taken smaller by a factor of 2 to find agreement with experiment.

In the present view the protein environment, besides determining the degree of energetic coincidence and the collision time $\tau$, has no pronounced specific influence on the rate (this is supported by the fact that deuteration of the sample ${ }^{2}$ has no influence on k).

The proposed energetic coincidence of $\left|\mathrm{P}^{*}\right\rangle$ and vibronically excited $\left|\mathrm{H}_{\mathrm{L}}{ }^{-}\right\rangle$is thought to be an evolutionary product to preserve electron flow in the $\mathrm{L}$ branch (in contrast to the $\mathrm{M}$ branch). The originally symmetric protein dimer has developed into the asymmetric form by gene duplication and mutations. There was a strong evolutionary pressure to preserve electron flow in the $\mathrm{L}$ branch but not in the $\mathrm{M}$ branch.?

This restriction of the electron flow to the $L$ branch is expected from the present model since the level of $\left|\mathrm{H}_{\mathrm{M}}{ }^{-}\right\rangle$is lower by $\Delta E_{\mathrm{LM}}$ $=0.1 \mathrm{eV}$ than the corresponding level of $\left|\mathrm{H}_{\mathrm{L}}{ }^{-}\right\rangle$(the value was obtained ${ }^{3}$ by considering the electrostatic interaction with the protein polar groups and asymmetric medium polarization interactions). Therefore, the above-mentioned coincidence is not given in the $\mathrm{M}$ branch. It follows from eq 1 and $1 \mathrm{a}$ that

$$
\frac{k_{\mathrm{L}}}{k_{\mathrm{M}}}=\frac{\left(2 \epsilon_{\mathrm{L}} / k_{\mathrm{B}} T\right) \arctan \left(k_{\mathrm{B}} T /\left(2 \epsilon_{\mathrm{L}}\right)\right)}{\left(2 \epsilon_{\mathrm{M}} / \Delta E_{\mathrm{LM}}\right)^{2}} \frac{\tau_{\mathrm{M}}}{\tau_{\mathrm{L}}}
$$

According to (4), assuming $\tau_{M}=\tau_{L}, \epsilon_{M}=\epsilon_{L}$, the rate at room temperature should be 86 times higher for the $L$ branch than for the $\mathrm{M}$ branch. In contrast, the unidirectionality cannot be explained by the energy difference of $0.1 \mathrm{eV}$ modifying the Franck-Condon factor.

The theory advanced in the present paper provides an interesting prediction. The proposed energetic coincidence in the $\mathrm{L}$ branch should be disturbed by environmental changes, and therefore changes in the medium should either diminish the rate or be ineffective. Indeed, the exchange of a polar glutamic acid residue (GLU 104) in the vicinity of $\mathrm{H}_{\mathrm{L}}$ by a nonpolar residue (GLN or LEU) diminishes the rate in $R b$. capsulatus by as much as $40 \%$ at room temperature. ${ }^{10}$ For a quantitative estimate of the change in rate by this substitution it must be considered that the carboxyl group of GLU104 is at the correct distance to the keto carbonyl group of ring $\mathrm{V}$ of $\mathrm{H}_{\mathrm{L}}$ to form a hydrogen bond."

For estimating the Coulomb energy between the carboxyl group and the $\pi$ electron in $\mathrm{H}_{\mathrm{L}}{ }^{-}$the carboxyl group is represented by point charges, $+0.38 \mathrm{e}(\mathrm{e}=$ elementary charge $)$ at the $\mathrm{H}$ atom, $-0.38 \mathrm{e}$ at the $\mathrm{O}$ atom of $\mathrm{C}-\mathrm{OH},+0.5 \mathrm{e}$ at the $\mathrm{C}$ atom, and -0.5 $e$ at the $\mathrm{O}$ atom of $\mathrm{C}=0 .{ }^{12}$ According to INDO calculations the density of the electron in $\mathrm{H}_{\mathrm{L}}{ }^{-}$at the $\mathrm{O}$ and $\mathrm{C}$ atom of the keto carbonyl group of ring $\mathrm{V}$ is $(0.18)^{2} \mathrm{e}$ and $(0.10)^{2} \mathrm{e}$, respectively. The electrostatic energy of these charges in the field of the carboxyl group thus obtained is $\Delta=0.031 \mathrm{eV}(\mathrm{O} \cdots \mathrm{H}$ and $\mathrm{O}-\mathrm{H}$ distance 1.9 and $1.0 \AA$, respectively). ${ }^{3,11}$ The carboxyl group is assumed

(9) This evolutionary concept was given in ref 8 and was later supported by X-ray and protein sequence analysis showing the presence of postulated pair of proteins and the genetic relation.'

(10) Bylina, E. J,; Kirmaier, Ch.; McDowell, L.; Holton, D.; Youvan, D. C. Nature 1988, 336, 182 .

(11) Michel, H.: Epp, O.: Deisenhofer, J. EMBO J. 1986, 5, 2445

(12) Weiner, P. A.; Kollman, D. A.; Case, U. C.; Singh, C.; Ohio, G.; Alogerma, S.; Profeta, Jr.; Weiner, P. J. Am. Chem. Soc. 1984, 106, 765. to be shielded by the protein environment so that $\Delta$ constitutes the energy by which the level of $\mathrm{H}_{\mathrm{L}}{ }^{-}$lifts when GLU104 is exchanged for GLN or LEU. The value $\Delta=0.05 \pm 0.02 \mathrm{eV}$ was given in ref 3 where shielding was neglected, thus leading to a slightly larger value.

Assuming $\tau$ is unaffected by the substitution, the ratio of rates $k_{\mathrm{L}}\left(\mathrm{GLN}\right.$ or LEU) $/ k_{\mathrm{L}}(\mathrm{GLU})$ is given by (see (1a))

$$
q=\frac{k_{\mathrm{L}}(\mathrm{GLN} \text { or LEU })}{k_{\mathrm{L}}(\mathrm{GLU})}=\frac{\arctan \frac{k_{\mathrm{B}} T+\Delta}{2 \epsilon}+\arctan \frac{k_{\mathrm{B}} T-\Delta}{2 \epsilon}}{2 \arctan \frac{k_{\mathrm{B}} T}{2 \epsilon}}
$$

and for the given values $T=300 \mathrm{~K}, \epsilon=3.2 \times 10^{-3} \mathrm{eV}$, and $\Delta$ $=0.03 \mathrm{eV}$ this gives $q=0.5$, corresponding to a $50 \%$ reduction in rate. This is in good agreement with the $40 \%$ reduction observed in ref 10 . With the simplifying assumptions considered here the rate should decrease with decreasing temperature in the case of the mutants and reach a 7 times smaller value at $T=0$, in contrast to the wild type where the rate increases with lowering temperature (Figure 1).

The exchange of GLU104 for GLN or LEU is accompanied by a hypsochromic shift of the absorption band of $\mathrm{H}_{L}$ by $6 \mathrm{~nm}$ corresponding to an increase in excitation energy of $0.026 \mathrm{eV}$ for GLN and by a slightly larger shift for LEU (band of $\mathrm{H}_{M}$ and $\mathrm{H}_{\mathrm{L}}$ unresolved).$^{10}$ A hypsochromic shift is expected since the electron density in the keto carbonyl group in ring $\mathrm{V}$ is increased in exciting $\mathrm{H}_{\mathrm{L}}$ from $(0.13)^{2}$ e to $(0.18)^{2}$ e $(\mathrm{O}$ atom $)$ and from $(0.03)^{2} \mathrm{e}$ to $(0.10)^{2}$ e (C atom). By summing up the Coulomb terms we find for the expected increase in excitation energy $0.02 \mathrm{eV}$. The good agreement with the measured value is a test supporting the model.

Note Added in Proof. In a recent paper by Holzapfel et al., ${ }^{13}$ the primary electron transfer in reaction centers of $R b$. sphaeroides was investigated with femtosecond time resolution, and the results were described by assuming that the electron in $\mathrm{P}^{*}$ is transferred to $\mathrm{B}_{\mathrm{L}}$ with time constant $3.5 \mathrm{ps}$ and from there to $\mathrm{H}_{\mathrm{L}}$ with time constant 0.9 ps. The slow population and fast depopulation of $B_{L}$ was assumed to account for the small transient absorption changes corresponding to a transient population of $\left(\mathrm{P}^{+} \mathrm{B}^{-} \mathrm{H}\right)$ of $16 \%$. In the present view the short-living transient is a state where $P, B_{L}$, and $H_{L}$ share the electron and the small absorption change is due to the fact that $B_{L}$ shares $16 \%$ only. According to the Appendix, eq A.4, this state is populated in about 0.5 ps after exciting $\mathrm{P}$, and according to Figure 1 it decays into the vibronic ground state of $\mathrm{H}_{\mathrm{L}}{ }^{-}$with a time constant of $3 \mathrm{ps}$. In the given range (delay time $t$ up to $20 \mathrm{ps)}$ this scheme describes the experimental results given in Figs. 1 and 2 in the paper by Holzapfel et al. The absorption rises within the flash duration of $100 \mathrm{fs}$. The subsequent absorption change can be described in the first picosecond by $\alpha\left(1-\exp (t / \bar{t})\right.$ ) with $\bar{t}=0.5 \mathrm{ps}, \alpha=3 \times 10^{-4}$ (case of Fig. 1 in ref 13), $\alpha=-20 \times 10^{-4}$ (case of Fig. 2a), and $\alpha=$ $40 \times 10^{-4}$ (case of Fig. 2b). The change after the first picosecond can be described accordingly with $\bar{t}=3 \mathrm{ps}, \alpha=-35 \times 10^{-4}$ (case of Fig. 1), $\alpha=50 \times 10^{-4}$ (case of Fig. 2a), and $\alpha=-8 \times 10^{-4}$ (case of Fig. 2b).

Acknowledgment. We are grateful to Dr. M. Plato for his INDO data and to the referee for drawing our attention to ref 10. Financial support by the Deutsche Forschungsgemeinschaft for E.v.K. is gratefully acknowledged.

\section{Appendix}

A system including three electronic levels is considered. There are the donor state $\left|\mathrm{P}^{*}\right\rangle$ of the excited special pair, the state of the accessory bacteriochlorophyll $\left|\mathrm{B}_{\mathrm{L}}{ }^{-}\right\rangle$, and the acceptor state $\left|\mathrm{H}_{\mathrm{L}}{ }^{-}\right\rangle$. The intermediate molecule is assumed to drastically improve the electron transfer from the donor to the acceptor, being

(13) Holzapfel, W.; Finkele, U.; Kaiser, W.; Oesterhelt, D.; Scherr, H.; Stilz, H. U.; Zinth, W. Chem. Phys. Lett. 1989, 160, 1. 
itself no kinetic intermediate acceptor. The Hamiltonian has the form

$$
\mathscr{H}_{0}=\left(\begin{array}{ccc}
E_{\mathrm{P}} & \epsilon_{\mathrm{PB}} & \epsilon_{\mathrm{PH}} \\
\epsilon_{\mathrm{PB}} & E_{\mathrm{B}^{-}} & \epsilon_{\mathrm{BH}} \\
\epsilon_{\mathrm{PH}} & \epsilon_{\mathrm{BH}} & E_{\mathrm{H}_{\mathrm{L}^{-}}}
\end{array}\right)
$$

Allthough the three-level system can be solved analytically by means of the Cardan formula, a more indirect way leads to less complicated results. It is assumed that the energy level $E_{\mathrm{p} *}$ of $\left|\mathrm{P}^{*}\right\rangle$ differs form the level $E_{\mathrm{H}_{\mathrm{L}}}$ - of vibronically excited $\left|\mathrm{H}_{\mathrm{L}}{ }^{-}\right\rangle$in the order of the coupling of these levels $\epsilon_{\mathrm{PH}}:\left|E_{\mathrm{P}^{*}}-E_{\mathrm{H}_{\mathrm{L}}}-\right| \sim \epsilon_{\mathrm{PH}}$. On the other hand the level $E_{\mathrm{B}_{\mathrm{L}}}$ of the intermediate $\mathrm{B}_{\mathrm{L}}$ lies considerably above $E_{\mathrm{P}^{*}}$ and $E_{\mathrm{H}_{\mathrm{L}}}$ - and this difference is larger than all transfer matrix elements: $\left(\left|E_{\mathrm{B}^{-}}-E_{\mathrm{H}_{1}}-\right| \sim\left|E_{\mathrm{B}_{\mathrm{L}^{-}}}-E_{\mathrm{P}^{*}}\right|\right) \gg$ $\left|\epsilon_{\mathrm{PB}}\right|,\left|\epsilon_{\mathrm{PH}}\right|,\left|\epsilon_{\mathrm{BH}}\right|$. In the resulting expressions only the lowest order terms in $\left|\epsilon_{\mathrm{PB}}\right| /\left|E_{\mathrm{B}_{\mathrm{L}^{-}}}-E_{\mathrm{P}^{*}}\right| \ll 1,\left|\epsilon_{\mathrm{BH}^{-}}\right| /\left|E_{\mathrm{B}_{\mathrm{L}^{-}}}-E_{\mathrm{H}_{\mathrm{L}^{-}}}-\right| \ll 1,\left|\epsilon_{\mathrm{PH}}\right| / \mid E_{\mathrm{B}_{\mathrm{L}^{-}}}$ $-E_{\mathrm{P}^{*} \mid} \mid \ll 1$, and $\left|\epsilon_{\mathrm{PH}}\right| /\left|E_{\mathrm{B}_{\mathrm{L}}-}-E_{\mathrm{H}_{L^{-}}}\right| \ll 1$ are retained. We are now looking for a transformation $\mathcal{A}$ which is able to transform our original Hamiltonian $\mathscr{H}_{0}$ into a different Hamiltonian $\mathscr{H}_{1}=$ $\mathcal{A}^{*} \mathscr{H}_{0} \mathcal{A}$ with zero matrix elements of $\left(\mathscr{H}_{1}\right)_{12}$ and $\left(\mathscr{H}_{1}\right)_{23}$ :

$\mathcal{A}=$

$$
\left(\begin{array}{lll}
+\cos (\alpha) & -\sin (\alpha) & 0 \\
+\sin (\alpha) & +\cos (\alpha) & 0 \\
0 & 0 & 1
\end{array}\right)\left(\begin{array}{lll}
1 & 0 & 0 \\
0 & +\cos (\beta) & -\sin (\beta) \\
0 & +\sin (\beta) & +\cos (\beta)
\end{array}\right)
$$

Because of $\left(\left|E_{\mathrm{B}^{-}}-E_{\mathrm{H}_{\mathrm{L}}}\right| \sim\left|E_{\mathrm{B}_{\mathrm{L}^{-}}}-E_{\mathrm{P}^{*}}\right|\right) \gg\left|\epsilon_{\mathrm{PB}}\right|,\left|\epsilon_{\mathrm{PH}}\right|$ the unitary matrix $\mathcal{A}$ can be expanded linearly with respect to $\alpha$ and $\beta$. In this case, $\alpha$ and $\beta$ can be expressed easily in terms of the matrix elements of $\mathcal{H}_{0}$ :

$$
\begin{aligned}
& \alpha \approx+\frac{\epsilon_{\mathrm{PB}}}{E_{\mathrm{P}^{*}}-E_{\mathrm{B}_{\mathrm{L}^{-}}}}-\frac{\epsilon_{\mathrm{PH}^{-}} \epsilon_{\mathrm{BH}}}{\left(E_{\mathrm{H}_{\mathrm{L}^{-}}}-E_{\mathrm{B}_{\mathrm{L}}}\right)\left(E_{\mathrm{P}^{*}}-E_{\mathrm{B}_{\mathrm{L}}}\right)}
\end{aligned}
$$

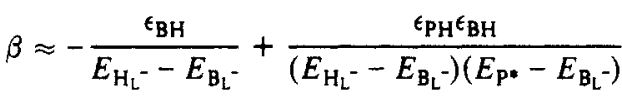

The transformed Hamiltonian $\mathscr{H}_{1}$ has the form

$$
\begin{aligned}
& H_{11}=\left(\mathcal{H}_{1}\right)_{11}=E_{\mathrm{P}^{*}}+\frac{\epsilon_{\mathrm{PB}}{ }^{2}}{E_{\mathrm{P}^{*}}-E_{\mathrm{B}_{1}}} \\
& H_{22}=\left(\mathscr{H}_{\mathrm{L}}\right)_{22}=E_{\mathrm{B}_{\mathrm{L}^{-}}}-\frac{\epsilon_{\mathrm{PB}}^{2}}{E_{\mathrm{P}^{*}}-E_{\mathrm{B}_{\mathrm{L}^{-}}}}-\frac{\epsilon_{\mathrm{BH}^{2}}}{E_{\mathrm{H}_{\mathrm{L}^{-}}}-E_{\mathrm{B}_{\mathrm{L}^{-}}}} \\
& H_{33}=\left(\mathscr{t}_{1}\right)_{33}=E_{\mathrm{H}^{-}}+\frac{\epsilon_{\mathrm{BH}^{2}}{ }^{2}}{E_{\mathrm{H}_{\mathrm{L}^{-}}-E_{\mathrm{B}^{-}}}} \\
& \epsilon=H_{13}=\left(\mathscr{H}_{1}\right)_{13}=\epsilon_{\mathrm{PH}}+\frac{\epsilon_{\mathrm{PB}} \epsilon_{\mathrm{BH}}}{E_{\mathrm{P}^{*}}-E_{\mathrm{B}_{\mathrm{L}^{-}}}}+\frac{\epsilon_{\mathrm{PB}} \epsilon_{\mathrm{BH}}}{E_{\mathrm{H}_{\mathrm{L}^{-}}-E_{\mathrm{B}_{\mathrm{L}^{-}}}}}
\end{aligned}
$$

The diagonalization of this Hamiltonian is equivalent to the straightforward two-level problem:

$$
\tilde{\mathcal{H}}=\left(\begin{array}{ll}
H_{11} & \epsilon \\
\epsilon & H_{33}
\end{array}\right)
$$

Let $\tilde{\mathcal{C}}$ be the unitary matrix that diagonalizes the Hamiltonian $\tilde{\mathcal{H}}$. From $\tilde{\mathcal{C}}$ the unitary matrix $\mathcal{C}$ can be constructed by diagonalizing $\mathscr{H}_{1}$. The most important term in the probability $P_{\mathrm{H}_{\mathrm{L}}}$ of finding the electron in the acceptor $\left|H_{\mathrm{L}}{ }^{-}\right\rangle$is given by

$$
P_{\mathrm{H}_{\mathrm{L}}}=\left|\left\langle\mathrm{P}^{*} ; t \mid \mathrm{H}_{\mathrm{L}}{ }^{-}\right\rangle\right|^{2}=\frac{4 \epsilon^{2}}{A^{2}} \sin ^{2}\left(\frac{A}{2 \hbar} t\right)
$$

with $A=\left[\left(H_{11}-H_{33}\right)^{2}+4 \epsilon^{2}\right]^{1 / 2}$. Similarly the probability of finding the electrons at $\mathrm{B}_{\mathrm{L}}$ can be calculated. The leading terms are given by

$$
P_{\mathrm{B}_{\mathrm{L}}}=\left|\left\langle\mathrm{P}^{*} ; t \mid \mathrm{B}_{\mathrm{L}}{ }^{-}\right\rangle\right|^{2}=\frac{4 \epsilon_{\mathrm{PB}}{ }^{2}}{B^{2}} \sin ^{2}\left(\frac{B}{2 \hbar} t\right)+\frac{4 \epsilon_{\mathrm{BH}^{2}}}{C^{2}} \sin ^{2}\left(\frac{C}{2 \hbar} t\right)
$$

with $B=\left[\left(H_{22}-H_{11}\right)^{2}+4 \epsilon_{\mathrm{PB}}{ }^{2}\right]^{1 / 2}$ and $C=\left[\left(H_{22}-H_{33}\right)^{2}+\right.$ $\left.4 \epsilon_{\mathrm{BH}}^{2}\right]^{1 / 2}$. For $B \gg \epsilon_{\mathrm{PB}}$ and $C \gg \epsilon_{\mathrm{BH}}$ one obtains $|C| \approx \| B|-| A||$.

\title{
Observation of ESR Spin Filp Satellite Lines of Trapped Hydrogen Atoms in Solld $\mathrm{H}_{2}$ at
}

\section{$4.2 \mathrm{~K}$}

\author{
Tetsuo Miyazaki, ${ }^{*}$ Nobuchika Iwata, Kenji Fueki, \\ Department of Synthetic Chemistry, Faculty of Engineering, Nagoya University, Chikusa-ku, Nagoya 464-01, \\ Japan
}

and Hirotomo Hase

Research Reactor Institute, Kyoto University, Kumatori-cho, Sennan-gun, Osaka 590-04, Japan (Received: March 20, 1989; In Final Form: August 30, 1989)

ESR spectra of $\mathrm{H}$ atoms, produced in $\gamma$-irradiated solid $\mathrm{H}_{2}$, were studied at $4.2 \mathrm{~K}$. Two main lines of the ESR spectra of $\mathrm{H}$ atoms that are separated by about $500 \mathrm{G}$ accompanied two weak satellite lines. Both satelite lines and main lines decrease with the same decay rate. In the $\mathrm{D}_{2}-\mathrm{H}_{2}$ mixtures, the satellite-line intensity depends upon the number of matrix protons. The spacing of the satellites from the main lines is equal to that of the NMR proton resonance frequency. It was concluded that the satellite lines were not ascribable to paired atoms but to spin flip lines due to an interaction of $\mathrm{H}$ atoms with matrix protons. The analysis of the spin flip lines and the main lines suggests that $\mathrm{H}$ atoms in solid $\mathrm{H}_{2}$ are trapped in the substitutional site.

\section{Introduction}

The role of quantum mechanical tunneling in reactions $\mathrm{H}_{2}$ $\left(D_{2}, H D\right)+H(D)$ has been one of the important problems in the

\footnotetext{
* Author to whom correspondence should be addressed.
}

theory of chemical kinetics. When hydrogen atoms are produced by $\gamma$-radiolysis of solid hydrogen at an ultralow temperature, the hydrogen atoms react with hydrogen molecules by tunneling. Miyazaki et al. ${ }^{1-5}$ have obtained the following results on the

(1) Miyazaki, T.; Lee, K. P. J. Phys. Chem. 1985, 90, 400. 